\title{
Two models with rescattering for high energy heavy ion collisions
}

\author{
H. Bøggild* and Ole Hansen ${ }^{\dagger}$ \\ University of Copenhagen, Niels Bohr Institute, Copenhagen, Denmark \\ T. J. Humanic $\ddagger$ \\ Department of Physics, Ohio State University, Columbus, Ohio, USA \\ (Received 11 February 2006; published 7 December 2006)
}

\begin{abstract}
The effects of hadronic rescattering in high energy relativistic $\mathrm{Au}+\mathrm{Au}$ collisions are studied using two very different models to describe the early stages of the collision. One model is based on a hadronic thermal picture and the other on a superposition of parton-parton collisions. Operationally, the output hadrons from each of these models are used as input to a hadronic rescattering calculation. The results of the rescattering calculations from each model are then compared with rapidity and transverse momentum distributions from the BNL Relativistic Heavy Ion Collider BRAHMS experiment. In spite of the different points of view of the two models of the initial stage, after rescattering, the observed differences between the models are mostly "washed out" and both models give observables that agree roughly with each other and with experimental data.
\end{abstract}

DOI: 10.1103/PhysRevC.74.064905

PACS number(s): 25.75.Dw, 25.75.Gz, 25.75.Ld

\section{INTRODUCTION}

The main goal of studying relativistic heavy ion collisions at the BNL Relativistic Heavy Ion Collider (RHIC) accelerator is to obtain information about the early stage of the collision when matter is at its most hot and dense state. Since experimentally one detects hadrons which have undergone final-state rescattering before decoupling from the collision, it is of interest to use model calculations to seek to disentangle the hadronic rescattering effect that tends to wash out the information about the early state of matter in which we are most interested. Such a rescattering calculation was carried out for RHIC collisions assuming a simple thermal-like model to describe the early stage of the collision [1-3], but since that thermal model was so simple, it proved difficult to interpret the results for the initial stage. The present work improves on the previous study in two ways: (i) a more elaborate thermal-like model is used for the initial stage, and (ii) a second initial-stage model based on a superposition of parton-parton collisions is also included in the study. The advantage of (i) is clearly to make the interpretation of the initial-stage results easier, and the advantage of (ii) is to compare the results of the thermal-like model with a model from a very different point of view, i.e., partonic, to see if after rescattering identifiable features of the different initial-stage models survive. We thus hope to address, at least for these two models, to what extent rescattering washes out the information about the initial stage of the collision. Our comparisons will be made with the hadronic observables rapidity and transverse momentum distributions, and these in turn will be compared with those extracted from the RHIC BRAHMS experiment $[4,5]$ as well as other RHIC experiments.

Sections II, III, and IV describe the thermal-like model, parton model, and rescattering calculation method, respectively.

\footnotetext{
*Email address: boggild@nbi.dk

†Email address: ohansen@nbi.dk

†Email address: humanic@mps.ohio-state.edu
}

Sections V and VI give results of coupling the rescattering calculation with the thermal and parton models, respectively, and of comparisons with experiment. Most comparisons are with data from the BRAHMS experiment because this experiment has particle identification also at forward rapidities. Section VII presents a discussion of the results.

\section{THE THERMAL-LIKE MODEL}

\section{A. Overview}

The thermal-like model that we use builds on the Bjorken picture of a high energy heavy ion reaction [6]. The two heavy ions pass through one another in a central collision, whereby both nuclei become highly excited and a color field of high energy density is created in the space between the two ions after the collision. Particles are produced, in part from the two original nuclei with a net-baryon number to ensure baryon conservation and in part from the region between the two ions, a region with a near-vanishing net-baryon content. It is assumed here that the produced particles may be described as originating from three Boltzmann source centers, corresponding to the two heavy ions and the energy field in between. The source centers are extended in rapidity space; in the present model, each is distributed over a Gaussianshaped rapidity probability density. All three source centers are assumed to have the same temperature parameter $T$, but they may contribute different numbers of particles. The created particles are assumed to have energy distributions that follow the Boltzmann distribution. The thermal model presented below thus creates for each type of particle three pools of four-momentum vectors, each pool distributed in rapidity around a source center. The differential distributions are then created by a weighted sum over contributions from the three source centers, where the weights $N_{C}$ are the number of four-momentum vectors (particles) from each center. Finally, the particles thus produced are allowed to rescatter as they emerge, see Sec. IV. 
It is well documented from previous work [7] that particle ratios, in principle integrated over the entire phase space, are described very well by thermal statistical ensembles; but thermal models that give insight into differential distributions in rapidity, transverse mass, or momentum are scarce. The purpose of the exercise presented here is to show such differential distributions, compare them with data, and obtain some understanding as to the extent to which such a description can reproduce the main features of the observations.

\section{B. Structure of the model}

A particle is characterized by an index $i$, standing for $\pi^{+}, K^{+}, p$, or $\bar{p}$, with mass $m=m(i)$. The particle is created by a Boltzmann-like source belonging to a source center $C$ with rapidity $y_{C}$. The source rapidity $y_{G}$ is chosen by a Monte Carlo routine as a deviation from $y_{C}$ via a Gaussian probability density distribution

$$
G\left(y_{G}, y_{C}\right)=\frac{1}{\sigma_{C} \sqrt{2 \pi}} \exp \left[-\left(y_{G}-y_{C}\right)^{2} / 2 \sigma_{C}^{2}\right],
$$

where $\sigma_{C}$ characterizes the width of the distribution around $y_{C}$. The laboratory rapidity of the source $y_{S}$ is

$$
y_{S}=y_{G}+y_{C} .
$$

The four-momentum of the particle $i$ is generated in the local source reference system, which moves with rapidity $y_{S}$ in the laboratory system, by choosing the polar and azimuthal angles, $\theta_{B}$ and $\phi_{B}$, such that the polar angle is taken from a constant distribution in $\cos \left(\theta_{B}\right)$ for $0<\theta_{B}<\pi$, and $\phi_{B}$ is evenly distributed from 0 to $2 \pi$. The energy of the particle in the local source system, $E_{i}$, is finally chosen according to a Boltzmann prescription,

$$
B\left(E_{i}, T\right)=\frac{E_{i} \sqrt{E_{i}^{2}-m(i)^{2}}}{T m(i)^{2} K_{2}(m(i) / T)} \exp \left(-E_{i} / T\right) .
$$

The temperature parameter $T$ is a global parameter used for all particle types, source centers, and local sources. $K_{2}$ is a modified Bessel function [8]. The four-momentum components $\left(E_{i}, p_{x}, p_{y}, p_{z}\right)$ following from the Monte Carlo choices are then Lorentz transformed to the laboratory system and the process started over again with a new choice of $y_{G}$.

Each particle thus originates with its four-momentum from its own local source reference system. If the width $\sigma_{C}=0$, the particles from source center $C$, will represent particles from a spherically symmetric Boltzmann source in the source-center reference system. The widths of the Gaussian rapidity distributions used below are quite broad, and the $T$ parameter does not represent a source temperature in the thermal sense, but rather a parameter that determines the hardness of the emitted momenta, and it is not a freeze-out temperature. A certain number of particles $N_{i, C}$ are generated from each source center, and the collection of four-vectors then constitutes the model data for particle species $i$. The multiplicity density distribution is then obtained as the sum of three source-center contributions

$$
d n_{i} / d y=N_{i, 0} F_{i, 0}(y)+N_{i,-} F_{i,-}(y)+N_{i,+} F_{i,+}(y),
$$

where $y$ is the laboratory rapidity, and $F_{i, C}(y)$ the probability density of finding an $i$ particle with laboratory rapidity $y$ originating from source center $C$. The products $N_{i, C} F_{i, C}(y)$ are the results of the Monte Carlo computation as described.

\section{Parameters of the model}

For a particle of species $i$, the model has ten parameters: temperature $T$, rapidities of the three source centers $y_{i,+}, y_{i, 0}$, and $y_{i,-}$, widths of the three Gaussian distributions $\sigma_{i,+}, \sigma_{i, 0}$, and $\sigma_{i,-}$, and number of particles from each source center $N_{i,+}, N_{i, 0}$, and $N_{i,-}$, where the subscripts,+ 0 , and - refer to the sequence in rapidity of the centers $C$. In this report, only symmetric collisions, $A+A$, are considered and four restrictions are imposed on the parameters,

$$
\begin{aligned}
y_{i, 0} & =0, \\
y_{i,+} & =-y_{i,-}, \\
\sigma_{i,+} & =\sigma_{i,-}, \\
N_{i,+} & =N_{i,-} .
\end{aligned}
$$

Initially $T=200 \mathrm{MeV}$ was tried, but for the main part of the paper $T=270 \mathrm{MeV}$ was used, as it gave a better account of the transverse spectra (Sec. V). The $N_{i, C}$ values are chosen for each $(i, C)$ combination. The ratio $N_{i,+} / N_{i, 0}$ has a decisive influence on the shape of the predicted rapidity density distribution, $d n / d y$, and on the slopes of the transverse spectra. The same holds true for the $y_{i,+}, \sigma_{i,+}$, and $\sigma_{i, 0}$ values. The parameters were determined by asking for a reasonable agreement with the proton $d n / d y$ distribution for $\mathrm{Au}+\mathrm{Au}$ at $\sqrt{s}=200 \mathrm{GeV}$ per nucleon as measured by the BRAHMS Collaboration [4], in the expectation that the $y_{p,+}, \sigma_{p,+}$, and $\sigma_{p, 0}$ found in this way could be used for all particle species $i$, an expectation that was fulfilled, so the index $i$ can be dropped from these three quantities.

The BRAHMS proton data show that $y_{+}$is larger than 3.0, but they do not fix the value because of the limited rapidity coverage of the experiment. The value used here of $y_{+}=3.5$ is a reasonable value, particularly when the measured distribution of net protons is also considered, but the best value might be larger. The shape of a predicted $d n / d y$ distribution is changed moderately, but not drastically, by introducing the rescattering routines (see later), a fact that cuts down the computing time for obtaining the needed parameters, because the fitting could be done quite reliably without the rescattering. In fact, the computing time with rescattering would have rendered the fitting process impractical.

The actual fitting was made by varying the Gaussian $\sigma$ 's and the proton $N_{i,+}$ and $N_{i, 0}$ in a trial-and-error way. The final values, kept constant for the remaining use of the model here, are given in Table I. They do not necessarily represent a best fit (which was never sought after), but they do represent a reasonable fit. A comparison between the model and the BRAHMS proton data [4] for $d n / d y$ is shown in the bottom part of Fig. 1. For all other particles $\left(\bar{p}, \pi^{+}\right.$, and $\left.K^{+}\right)$, only the corresponding $N_{i,+}$ and $N_{i, 0}$ values were adjusted to give the measured ratio between $d n / d y$ at $y=0$ and at the highest 
TABLE I. Fit parameters for $T=$ 200 and $270 \mathrm{MeV}$.

\begin{tabular}{lcc}
\hline \hline Particle & Quantity & Values \\
\hline All & $y_{0}$ & 0.00 \\
All & $y_{+}$ & 3.50 \\
All & $\sigma_{0}$ & 1.50 \\
All & $\sigma_{+}$ & 2.00 \\
$p$ & $N_{i,+} / N_{i, 0}$ & 0.95 \\
$\bar{p}$ & & 0.18 \\
$\pi^{+}$ & & 0.40 \\
$\mathrm{~K}^{+}$ & & 0.45 \\
\hline \hline
\end{tabular}

value of $y$ at which there were data [5] for the particle type in question. The $N_{i,+} / N_{i, 0}$ values obtained are shown in Table I, and comparisons to the data in Fig. 1. Transverse spectra may be constructed from the model data by selecting a rapidity interval and sampling the number of particles as a function of $m_{t}$ or $p_{t}$. For a given species, the spectrum is again made as a weighted sum of contributions from the three source centers,

$$
E d^{3} n_{i} / d p^{3}=N_{i, 0} f_{i, 0}\left(m_{t}\right)+N_{i,+} f_{i,+}\left(m_{t}\right)+N_{i,-} f_{i,-}\left(m_{t}\right),
$$

where the $f$ functions are the probability densities to find a particle in the chosen rapidity bin with transverse mass $m_{t}$, and the integral of an $f$ function over $m_{t}$ equals the value of the

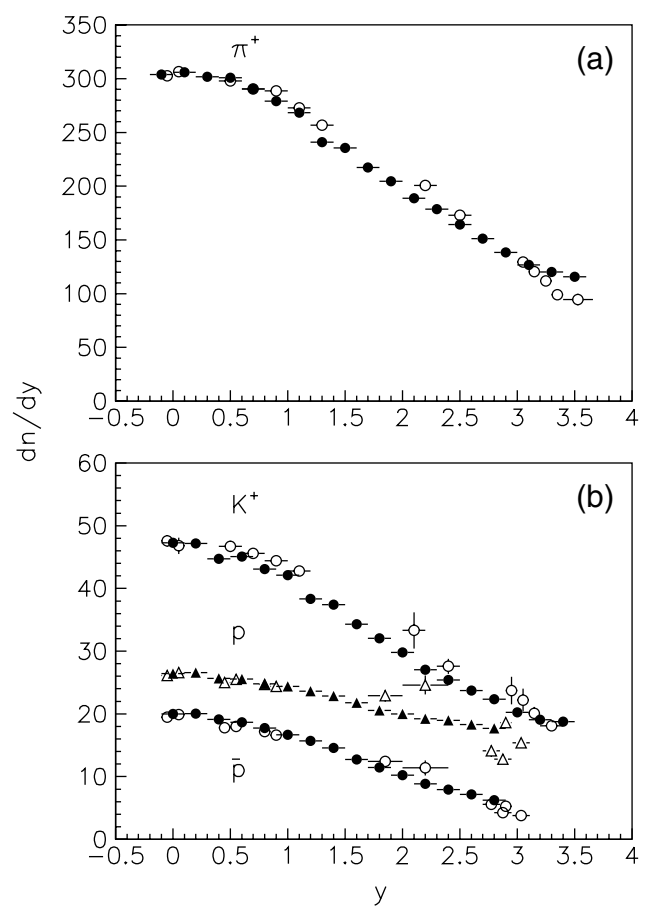

FIG. 1. Rapidity density $d n / d y$ plotted against center-of-mass rapidity for $\pi^{+}, K^{+}, p$, and $\bar{p}$. Open symbols designate data from BRAHMS [4,5]; black symbols are used for thermal model data, $T=200 \mathrm{MeV}$. The normalizations of the model prediction for each particle are made to make the $d n / d y$ near $y=0$ agree with the data, while the shapes of the model results emerge from the parameter choices as explained in the text.
$F$ function introduced in Eq. (4) for the chosen $y$. The shapes of the model spectra are more sensitive to the rescattering routines, and a discussion of spectra and comparisons with measurements are deferred to later in the paper. The thermallike model as presented here has no overall normalization, not even for a specific particle type. Normalizations were used only when model spectra were compared against data and are then arbitrary, so agreement or disagreement between model and data pertain solely to the spectral shapes.

\section{THE PARTONIC CLUSTER MODEL}

\section{A. Overview}

The "partonic cluster model" used in this study consists of two parts. One is a simple model for $p p$, or rather nucleonnucleon collisions, each of which consists of a partonic collision leading to two clusters and two ongoing wounded nucleons. The second part is a model for $A A$ collisions, which depending on the value of the impact parameter, leads to a number of such binary partonic collisions with onwardmoving wounded nucleons, allowed to reinteract. In both models, energy and momentum are approximately conserved by keeping track of the energy used in each step.

\section{B. The $p p$ Model}

In the present approach, each nucleon-nucleon collision has a hard scattering between two partons, leading to a system of two back-to-back $e^{+} e^{-}$-like clusters (jets) with multiplicities as a function of center-of-mass (c.m.) energy and particle composition obtained from $e^{+} e^{-}$data [9]. The collision takes up a certain fraction $x$ of the energy of each of the incoming nucleons, picked from an "effective" structure function distribution. A very simple probability distribution function is found adequate, i.e., $d W / d x=2(1-x)$, such that on average one-third of the nucleon's incoming energy participates in the hard scattering. Since the two parton energies are independent, the c.m. for the two-cluster system is not the overall c.m., but different from event to event. The remaining forward-going nucleon systems are considered excited systems, each of which fragments into one nucleon and one $e^{+} e^{-}$-like cluster (jet). Here it is assumed that on average half the energy is taken by the nucleon, i.e., a flat $x$ spectrum is used for the nucleon energy. Figure 2 shows how this model reproduces the mean charged multiplicity in $p p$ collisions in the energy range from 10 to $1000 \mathrm{GeV}$.

In the cluster fragmentation, the multiplicity of each cluster is taken from a negative binomial distribution of order $k$ of the quantity $n$ with average value $\langle n\rangle$,

$$
P(n,\langle n\rangle ; k)=\frac{(n+k-1) !}{n !(k-1) !} \frac{1}{(1+k /\langle n\rangle)^{n}} \frac{1}{(1+\langle n\rangle / k)^{k}},
$$

with the value of $k$ varying (decreasing) as a function of $\sqrt{s}$ and the longitudinal $x$ distribution is subsequently determined by one-dimensional longitudinal phase space.

The transverse momentum is generated by a procedure that accounts for the effect of gluon bremsstrahlung. This is done by giving the mean $p_{t}$ two components, one which is assumed 


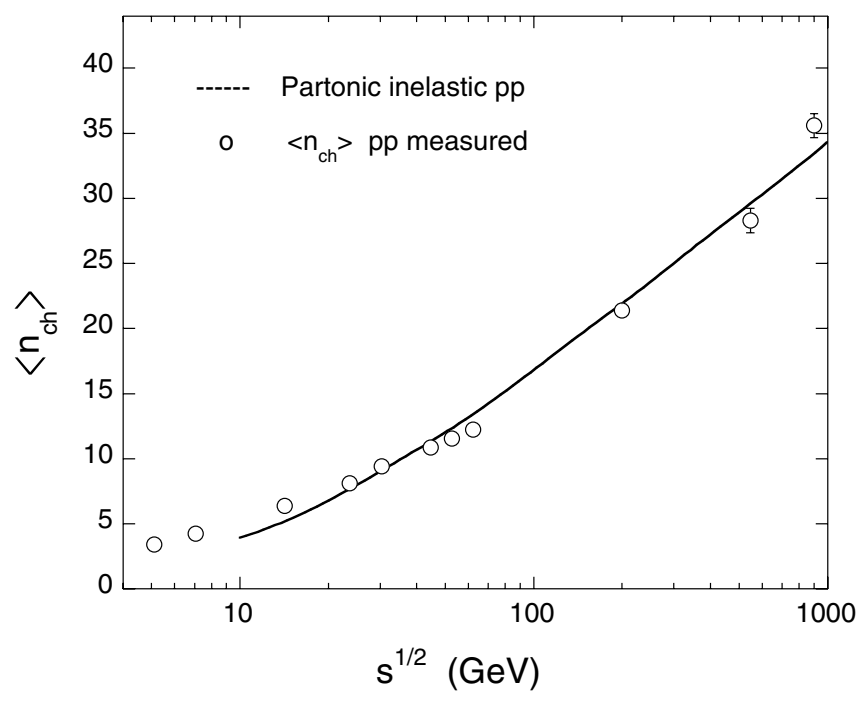

FIG. 2. Comparison of measured charged multiplicity in $p p$ collisions [9] and predictions from the partonic model.

constant $p_{t}^{\text {out }}$, and one increasing linearly with $\sqrt{s}, p_{t}^{\text {in }}$. This is in good qualitative agreement with $e^{+} e^{-}$data, Ref. [10]. We find that adding (in quadrature) the transverse momenta from two independent $m_{t}$ distributions with inverse slopes of $0.090 \mathrm{GeV} / c^{2}$ for $p_{t}^{\text {out }}$ and $0.180+(\sqrt{s}-20) 0.001$ for $p_{t}^{\text {in }}$ gives a good agreement between the model and $p p$ data, see Fig. 3. Note that the effective parton-parton scattering angle distribution (see below) is also involved in generating the $p_{t}$ distributions shown. For forward nucleons, a $p_{t}$ distribution with a negative inverse slope of $0.175 \mathrm{GeV} / c$ is used. The next step is to obtain an effective parton-parton scattering angle distribution for the two-cluster system. In this work, an angular distribution from Perkins [11] is used:

$$
d \sigma / d \cos \theta=\text { const } \times\left(3+\cos ^{2} \theta\right)^{3} /\left(1-\cos ^{2} \theta\right)^{2} .
$$

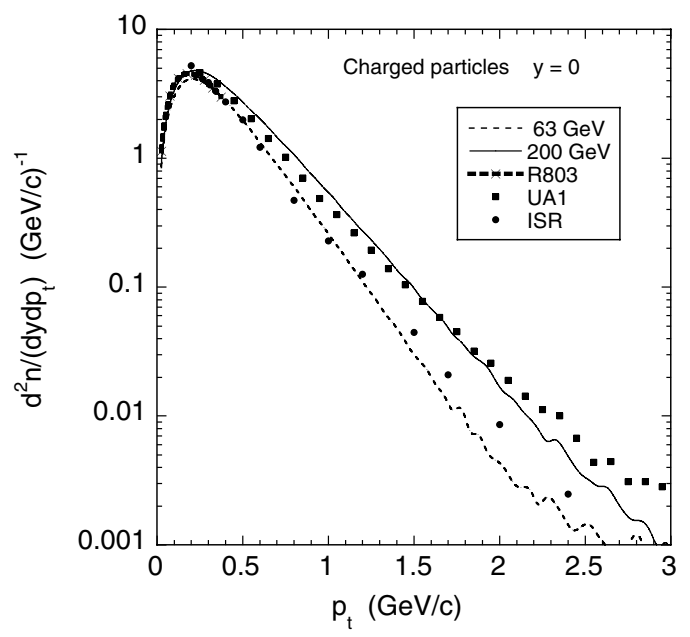

FIG. 3. $p_{t}$ distributions at $y=0$ from $p p$ collisions. The squares indicate UA1 data [12] at $\sqrt{s}=200 \mathrm{GeV}$. The stippled curve labeled $\mathrm{R} 803$ (seen at low $p_{t}$ only) and the filled circles are ISR results [12]. The dashed curves show partonic model results at $63 \mathrm{GeV}$ (lower) and $200 \mathrm{GeV}$ (upper).

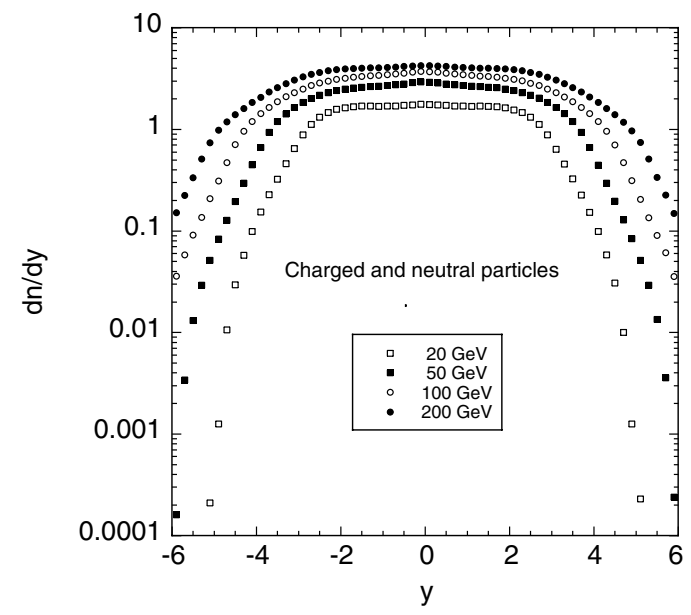

FIG. 4. Rapidity distributions in $p p$ collisions at c.m. energies of $20,50,100$, and $200 \mathrm{GeV}$ from the partonic model.

An angle is found above a cutoff, $p_{t, \text { cut }}$, following the above probability distribution, and the corresponding cluster $p_{t}$ is calculated. Finally this $p_{t}$ is reduced by the cutoff value: $p_{t, \text { cluster }}=\sqrt{p_{t}^{2}-p_{t, \text { cut }}^{2}}$. The value of the $p_{t}$ cutoff has direct influence on the normalization of the $p_{t}$ spectra at $p_{t}>$ $1 \mathrm{GeV} / c$. The value of $0.3 \mathrm{GeV} / c$ is found to lead to good agreement with $p p$ data for $\sqrt{s}<200 \mathrm{GeV}$.

It should be noted that once the c.m. energy in the parton-parton subsystem is chosen the angular distribution and fragmentation do not depend on the overall nucleon-nucleon (or $A A$ ) collision energy. Figures 3 and 4 show the $p_{t}$ and rapidity distributions obtained at different energies and illustrates in the $p_{t}$ case that acceptable agreement with the data is obtained.

\section{The $A A$ model}

The $A A$ model is inspired by the work of Jackson and Boggild [13] and subsequent work [14]. Note that the partonic model presented below also resembles in several respects the HIJING model of Wang and Gyulassy [15]. Each AA collision, with specified impact parameter, involves a certain number of binary collisions $N_{b}$ and participants $N_{p}$. The ratio of these numbers is the average number of collisions each struck nucleon experiences, $N_{c}=N_{b} / N_{p}$. If we now let a train of $N_{c}$ nucleons from one nucleus collide with a similar train from the other nucleus and do this $N_{p} / N_{c}$ times, we get the right number of binary collisions. In each train-train collision, the procedure is the following using the above-described parton-inspired $p p$ model:

(i) The two $e^{+} e^{-}$-like clusters escape the collision and fragment.

(ii) The forward wounded nucleon reinteracts with reduced energy.

(iii) At the end of the train, the wounded nucleon fragments as in the $p p$ model.

In this way, a collision of a train of five nucleons against another train of five nucleons will produce ten wounded 


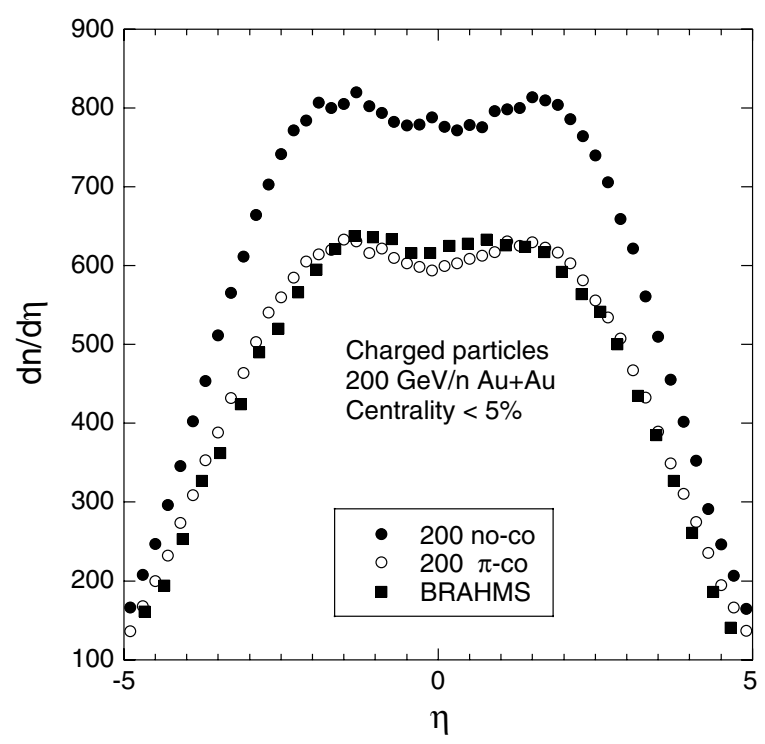

FIG. 5. Pseudorapidity distributions for central $\mathrm{Au}+\mathrm{Au}$ collisions at a c.m. energy of $200 \mathrm{GeV}$ compared with data from Brahms [16]. Full circles mark calculations at $200 \mathrm{GeV}$ without pion coalescence (see text, Sec. III D); open circles, calculations with pion coalescence.

nucleons with successively reduced energy and $25 e^{+} e^{-}$-like two-cluster systems, in total leading to 50 (binary) +10 (fragment) clusters +10 nucleons.

Figure 5 shows results of the model for central collisions of gold on gold, i.e., the pseudorapidity distributions before and after coalescence (see below) compared with BRAHMS data [16].

\section{A modification of the model}

From Fig. 5 it is clear that the $A A$ model leads to charged particle multiplicities which are too high by $30 \%-40 \%$. Simple lowering of the multiplicity in the nucleon-nucleon model does not work because energy conservation then forces particles in the forward direction to take up the missing energy. This leads to a forward peaked rapidity distribution. To lower the multiplicity while maintaining energy-momentum conservation and, at the same time, the shape of the rapidity distribution, an ad hoc procedure of pion coalescing, where two close-by pions merges into one, is adopted; the procedure used is the following:

(i) The $Q_{\text {inv }}^{2}$ of all pion pairs is calculated, where $Q_{\text {inv }}^{2}=$ $\left(E_{1}-E_{2}\right)^{2}-\left(p_{x 1}-p_{x 2}\right)^{2}-\left(p_{y 1}-p_{y 2}\right)^{2}-\left(p_{z 1}-p_{z 2}\right)^{2}$, and $E$ designates the total energy of the pion.

(ii) Pion pairs with $-Q_{\text {inv }}^{2}$ lower than a certain cutoff are coalesced.

(iii) This procedure is only carried out once.

It turns out that a cutoff at $-Q_{\text {inv }}^{2}=0.04^{2} \mathrm{GeV}^{2}$ leads to a reasonable reduction of the charged particle multiplicities and, at the same time, to a good agreement with observed rapidity distributions as demonstrated for BRAHMS in Fig. 5. The assumption behind the above phenomenological procedure is that nearby pions are for some time after creation still overlapping and can act together. As further discussed below, the price paid is a hardening of the pion $p_{t}$ distribution where the density is high.

\section{HADRONIC RESCATTERING CALCULATION}

The rescattering model calculational method used is similar to that employed in previous calculations for lower CERN Super Proton Synchrotron (SPS) energies and RHIC studies [1]. Rescattering is simulated with a semiclassical Monte Carlo calculation which assumes strong binary collisions between hadrons. The Monte Carlo calculation is carried out in three stages: (1) initialization and hadronization, (2) rescattering and freeze-out, and (3) calculation of experimental observables. Relativistic kinematics is used throughout.

The hadronization model inputs momentum vectors from the thermal model or partonic cluster model (both described above) and employs simple parametrizations to describe the initial space-time geometry of the hadrons similar to that used by Herrmann and Bertsch [17]. The initial space-time geometry of the hadrons for $b=0 \mathrm{fm}$ (i.e., zero impact parameter or central collisions) is parametrized as having cylindrical symmetry with respect to the beam axis. The transverse particle density dependence is assumed to be that of a projected uniform sphere of radius equal to the projectile radius $R\left(R=r_{0} A^{1 / 3}\right.$, where $r_{0}=1.12 \mathrm{fm}$ and $A$ is the atomic mass number of the projectile). The initial transverse coordinates of a given particle, i.e., $x_{\text {had }}$ and $y_{\text {had }}$, are thus determined according to this distribution. The longitudinal particle hadronization position $\left(z_{\text {had }}\right)$ and time $\left(t_{\text {had }}\right)$ are determined by the relativistic equations [6],

$$
z_{\text {had }}=\tau_{\text {had }} \sinh y_{i}, \quad t_{\text {had }}=\tau_{\text {had }} \cosh y_{i},
$$

where $y_{i}$ is the initial particle rapidity and $\tau_{\text {had }}$ is the hadronization proper time. Thus, the space-time hadronization model has one free parameter to extract from experiment: $\tau_{\text {had }}$. Although only pions, kaons, and nucleons are input from the thermal model as the initial particle types for the rescattering calculation, other types of hadrons can be produced during rescattering. In all, the hadrons included in the calculation are pions, kaons, nucleons, and lambdas $(\pi, K, N$, and $\Lambda$ ), and the $\rho, \omega, \eta, \eta^{*}, \phi, \Delta$, and $K^{*}$ resonances. For simplicity, the calculation is isospin averaged (e.g., no distinction is made between the pion charge states).

The second stage in the calculation is rescattering which finishes with the freeze-out and decay of all particles. Starting from the initial stage $(t=0 \mathrm{fm} / c)$, the positions of all particles are allowed to evolve in time in small time steps $(\Delta t=$ $0.1 \mathrm{fm} / c$ ) according to their initial momenta. At each time step, each particle is checked to see (a) if it has hadronized ( $\left.t>t_{\text {had }}\right)$, (b) if it decays, and (c) if it is sufficiently close to another particle to scatter with it. Isospin-averaged $s$ - and $p$-wave cross sections for meson scattering are obtained from Prakash et al. [18]. The calculation is carried out to 100 $\mathrm{fm} / c$, although most of the rescattering finishes by about $50 \mathrm{fm} / c$. The rescattering calculation is described in more detail elsewhere $[2,19]$. The validity of the numerical methods used in the rescattering code have recently been studied and verified [3]. 
In the last stage of the calculation, the freeze-out and decay momenta and space-times are used to produce observables such as pion, kaon, and nucleon multiplicities and transverse momentum and rapidity distributions. The values of the initial pion, kaon, and nucleon multiplicities, overall temperature, and hadronization proper time are all constrained to give observables that agree with available measured hadronic observables. As a cross-check on this, the total energy from the calculation is determined and compared with the RHIC c.m. energy of $\sqrt{s}=200 \mathrm{GeV} /$ nucleon-pair to see that they are in reasonable agreement. When the rescattering model was applied to partonic model data, each partonic model event was fully normalized and consisted of roughly 5300 pions $\left(\pi^{+}+\pi^{0}+\pi^{-}\right), 1000$ kaons $\left(K^{+}+K^{-}+K^{0}+\bar{K}^{0}\right)$, and 800 nucleons $(p+\bar{p}+n+\bar{n})$, which agrees well with measurements (see,e.g., Figs. 4 and 5 of Ref. [20]). The thermal model is unnormalized; events were created with about 5000 pions, 600 kaons, and 750 nucleons, and these events form the basis of the results shown below. Rescattering calculations were also performed for events with 1000 kaons rather than 600 , and the only change was in the overall multiplicity, while the spectral shapes were essentially unchanged. The hadronization parameter $\tau_{\text {had }}=1 \mathrm{fm} / c$ was used for all calculations, the same value as used in a previous application to SPS data for $\mathrm{Pb}+\mathrm{Pb}$ collisions [19].

\section{RESULTS FROM THE THERMAL MODEL WITH RESCATTERING}

\section{A. $d n / d y$ with and without rescattering}

This section demonstrates the changes in the rapidity density distributions caused by rescattering. The rescattering routine was run event by event, and 20 events made up the total final event pool, which was analyzed into $d n / d y$ and invariant cross section distributions. The thermal model sometimes produces particles with very large rapidities (e.g., $|y| \geqslant 10$ ) in the forward and backward directions. These particles do not have a counterpart in a collision situation at any existing accelerator, so all particles with $|y| \geqslant 6.5$ were disregarded in the rescattering calculation. $y=6.5$ is the beam rapidity at RHIC for the data used in the subsequent comparisons (Sec. V C). Such high rapidity particles constituted about 9\% of the four-vectors generated by the thermal model, and after their removal, the total energy of the remaining particles in a $T=270 \mathrm{MeV}$ event was close to the total energy in a $\sqrt{s}=200 \mathrm{GeV} /$ nucleon-pair $\mathrm{Au}+\mathrm{Au}$ collision. The resulting $d n / d y$ distributions at a temperature of $T=200 \mathrm{MeV}$ for pions, kaons, and nucleons are shown in Fig. 6, where open circles denote the distributions before rescattering and black circles after rescattering. Figure 7 shows results at $T=$ $270 \mathrm{MeV}$. In all cases, the influence of the rescattering is finite and rather small. For nucleons, the $d n / d y$ with rescattering is lower by less than $5 \%$ at small $y$ than without rescattering, and higher by a similar amount at high rapidities. Kaons are changed less and in a similar way, and the pions are still less changed. Also the changes in going from 200 to $270 \mathrm{MeV}$ are quite small.

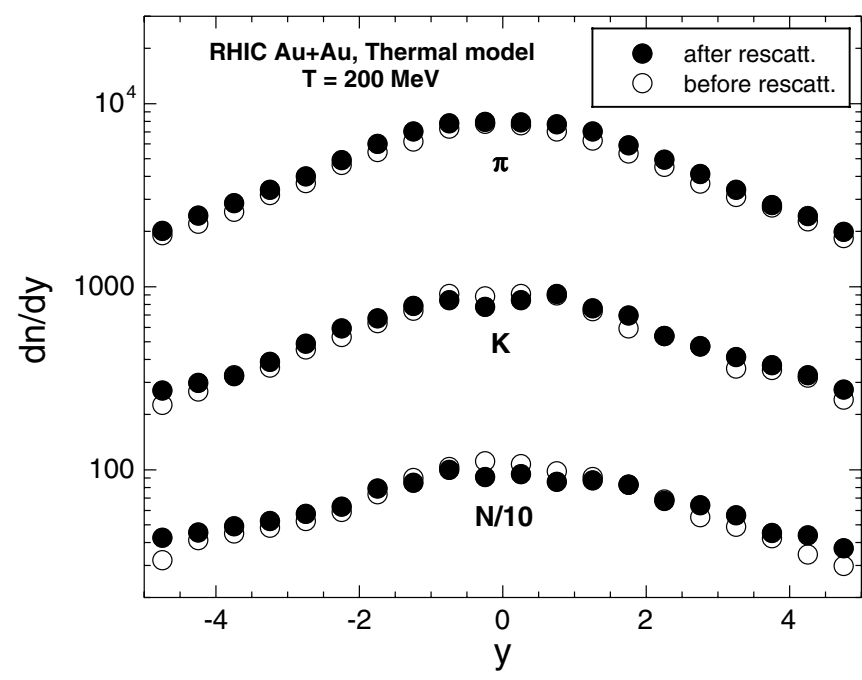

FIG. 6. Rapidity distributions from the thermal model with $T=$ $200 \mathrm{MeV}$, before and after rescattering. N/10 stands for nucleon multiplicity divided by 10 . The total multiplicities of each particle type are different from those in Fig. 1, and correspond to the multiplicities given in Sec. VI of the text.

\section{B. Transverse spectra with and without rescattering}

The invariant differential cross sections are analyzed as functions of either transverse momentum $p_{t}$ or transverse mass $m_{t}=\sqrt{p_{t}^{2}+m^{2}}$, that is,

$$
E \frac{d^{3} n}{d p^{3}}=\frac{d^{2} n}{2 \pi m_{t} d m_{t} d y}=\frac{d^{2} n}{2 \pi p_{t} d p_{t} d y},
$$

where the multiplicity is denoted by $n$.

For an isotropic Boltzmann source, the shape of the invariant $m_{t}$ spectrum reflects the temperature $T$ as

$$
\begin{aligned}
\frac{1}{2 \pi m_{t}} \frac{d^{2} n}{d m_{t} d y}= & N_{\mathrm{Bol}} m_{t} \cosh \left(y-y_{C}\right) \\
& \times \exp \left(-\frac{m_{t}}{T / \cosh \left(y-y_{C}\right)}\right),
\end{aligned}
$$

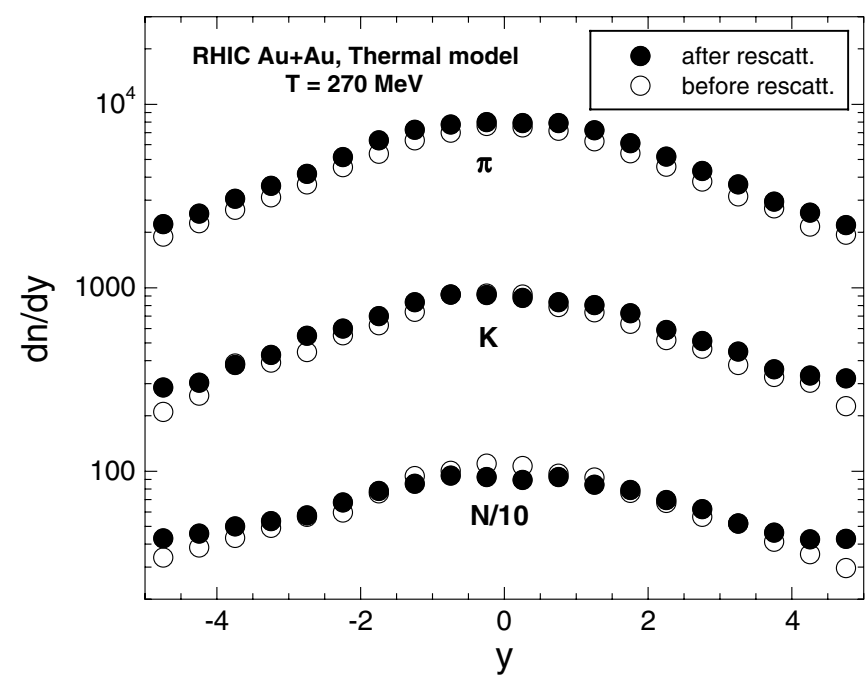

FIG. 7. Same as Fig. 6, but for $T=270 \mathrm{MeV}$. 


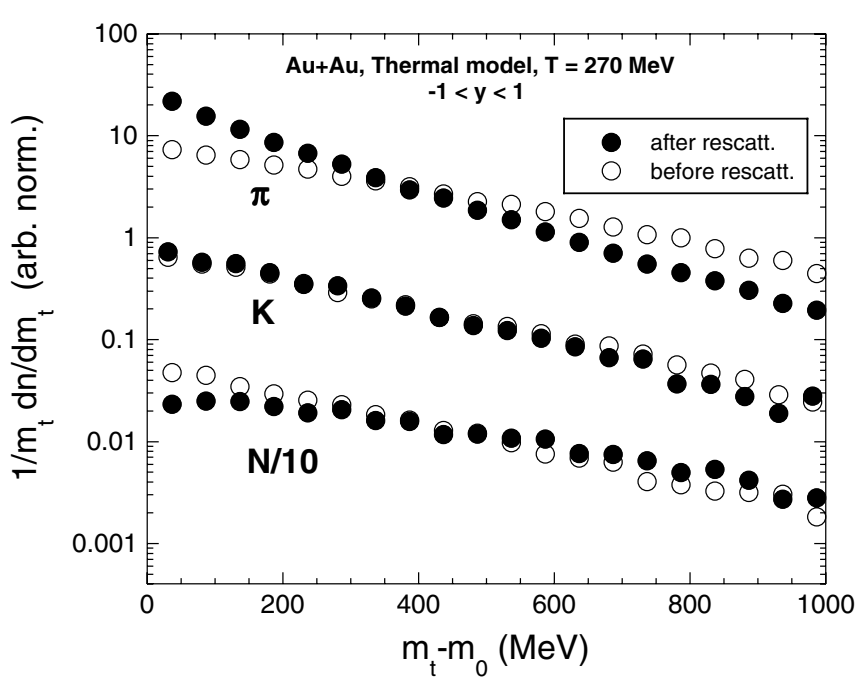

FIG. 8. Transverse mass spectra for $T=270 \mathrm{MeV}$ collected for rapidities around zero, before and after rescattering. $N / 10$ denotes nucleon multiplicity divided by 10 .

where $y_{C}$ is the rapidity of the source and $N_{\text {Bol }}$ the usual Boltzmann normalization [see also Eq. (3)]. The present thermal-like model represents the sum over many singleparticle sources, centered around three source centers, and will not show spectra following Eq. (13), nor the $1 / \cosh \left(y-y_{C}\right)$ dependence of the apparent temperature. Nonetheless, the $m_{t}$ spectra are nearly exponential, as in Eq. (13), and they exhibit an inverse slope that varies with rapidity in a way that also depends on particle mass, a feature that is different from the single spherical Boltzmann source, where the mass only enters explicitly in the normalization.

Figures 8 and 9 show the $m_{t}$ spectra before and after rescattering at $T=270 \mathrm{MeV}$ for rapidity zero and for rapidities near 3 , respectively. The rescattering produces a steeper fall off with $m_{t}$ for pions at both rapidity zero and 3.35; the rescattering, so to speak, cools the pions. Kaons are influenced by the rescattering in a similar way, but to

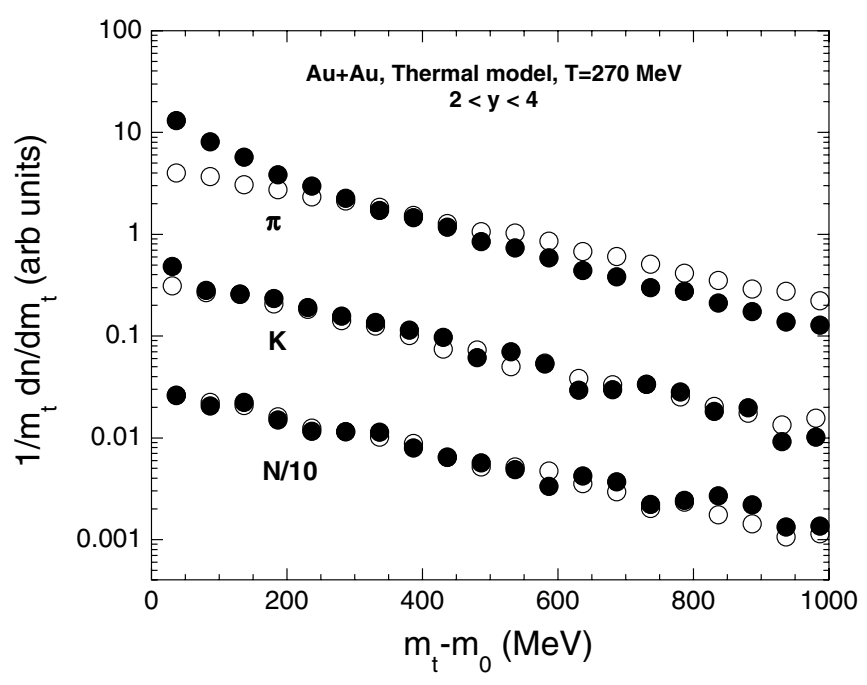

FIG. 9. Same as Fig. 8, but for rapidities of around 3.
TABLE II. Inverse slopes (MeV) thermal $270 \mathrm{MeV}$.

\begin{tabular}{lccc}
\hline \hline Particle & Rapidity & $\begin{array}{c}\text { Inverse slope } \\
\text { with rescatt }\end{array}$ & $\begin{array}{c}\text { Inverse slope } \\
\text { w/o rescatt }\end{array}$ \\
\hline$\pi$ & 0 & $195 \pm 1$ & $351 \pm 3$ \\
$K$ & & $267 \pm 5$ & $300 \pm 6$ \\
$N$ & & $449 \pm 1$ & $301 \pm 6$ \\
$\pi$ & 3 & $195 \pm 2$ & $326 \pm 4$ \\
$K$ & & $259 \pm 7$ & $289 \pm 9$ \\
$N$ & & $313 \pm 9$ & $293 \pm 8$ \\
\hline \hline
\end{tabular}

a smaller degree; while the nucleon spectrum becomes less steep in its fall off with $m_{t}$ at midrapidity, the nucleons get heated by the collisions with pions. At $y=3$, the nucleon spectrum is not changed by the rescattering. The inverse slopes are quantified in Table II, with the help of exponential fits to the spectra of Figs. 8 and 9, where the fitting ranges correspond to the $m_{t}$ ranges in the figures for each spectrum. The inverse slopes change with rapidity in a distinct way for each particle type, reflecting the relative weighting of the contributions from the middle and forward-backward source centers. The inverse slopes with rescattering also increase markedly with particle mass, a feature normally taken to be indicative of flow, something that has not been introduced explicitly in the model.

\section{Comparison with experimental data}

The rescattering calculations, as discussed above, have little influence on the model predictions for $d n / d y$, and the increase in temperature from 200 to $270 \mathrm{MeV}$ also has no marked effect on the model results with rescattering. Therefore, the agreement with data is similar to that shown in Fig. 1. The figure shows protons and antiprotons separately and the comparison after rescattering should rather be with nucleons; however, a comparison of proton plus antiproton data does not change the quality of the agreement.

The comparison between data and model results for the $p_{t}$ spectra is shown in Figs. 10 and 11 at $270 \mathrm{MeV}$. For $y=0$ (Fig. 10) the agreement between model and data is good, and at $y=3$ (Fig. 11) the agreement is less convincing but still reasonable. Thus the model can reproduce the data quite well at $T=270 \mathrm{Mev}$ at both rapidity intervals. It may be noted that the experimental $p_{t}$ spectra for protons and antiprotons exhibit nearly identical slopes [4] so the comparison of nucleon spectra from the model calculations to proton data is valid.

\section{RESULTS FROM THE PARTONIC MODEL WITH RESCATTERING}

\section{A. Comparison of results with and without rescattering}

Figure 12 shows the $d n / d y$ distributions for $\pi, K$, and nucleons from the partonic model (open circles) and after rescattering (black circles). The data cover 20 events as for the thermal model results. In agreement with the thermal model results, the rescattering changes the distributions by very little: they are slightly broadened. The $m_{t}$ spectra, however, are markedly influenced: rescattering makes the $\pi$ spectra steeper and flattens the $K$ and nucleon spectra, particularly 


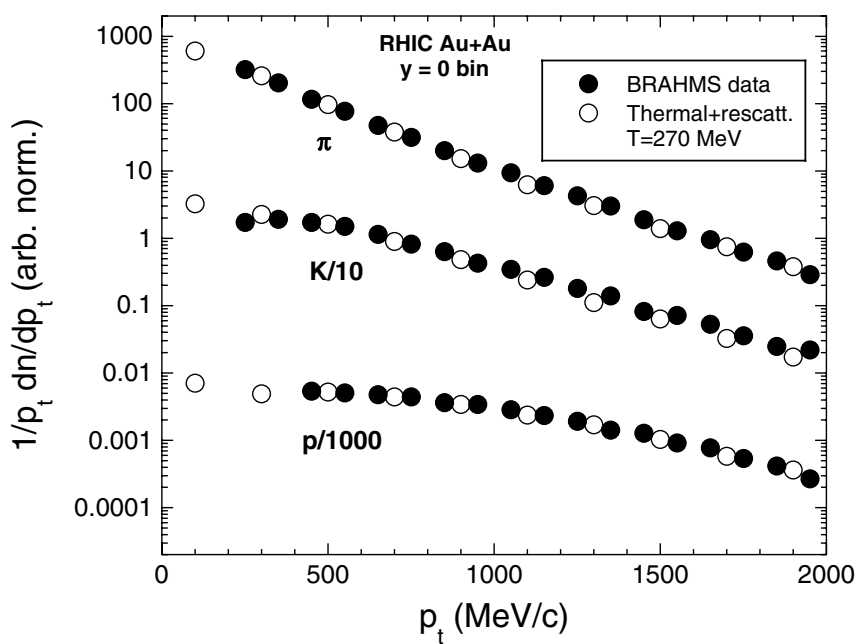

FIG. 10. Comparison of transverse momentum spectra for $T=$ $270 \mathrm{MeV}$ and data from BRAHMS [4,5] at midrapidity. $K$-meson multiplicities were divided by 10 ; proton data by 1000 . Model results are for nucleons rather than protons. Model data were matched to experiment at low $p_{t}$. Model results span rapidities from -1 to +1 ; data span the small rapidity interval of -0.1 to +0.1 .

at midrapidity. The effect for nucleons at midrapidity is somewhat stronger than for the thermal model, as demonstrated by the inverse slopes obtained from exponential fits quoted in Table III for the partonic model and in Table II for the thermal model. The changes are in the same directions as for the thermal model calculations.

The changes for nucleons caused by the rescattering are indeed quite dramatic, as is the cooling of the pions.

\section{B. Comparison to data}

The $d n / d y$ distributions after rescattering are compared to the data and thermal model for $\pi, K$, and nucleons in Fig. 13, where the data are for protons rather than for nucleons. The

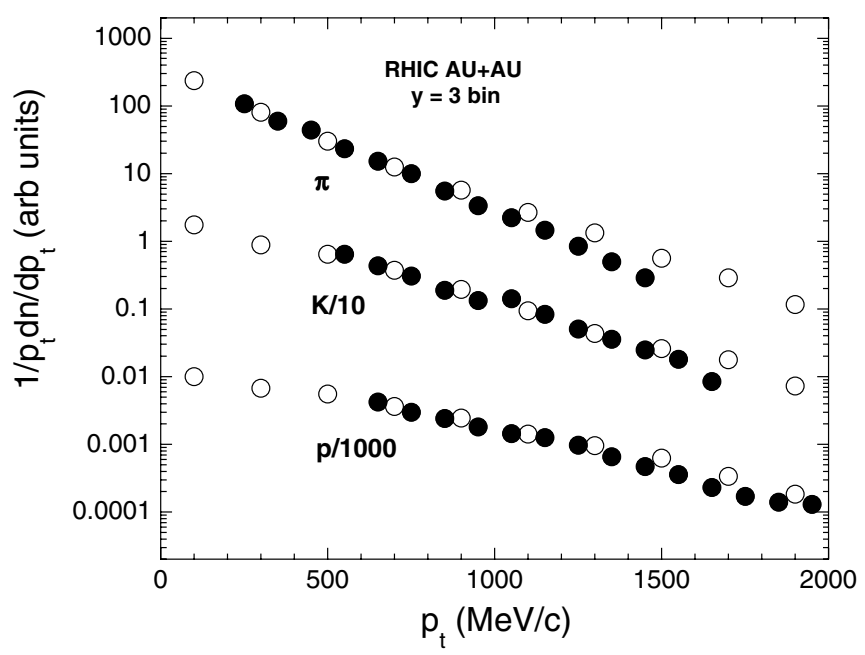

FIG. 11. Same as Fig. 10, but for rapidities of about 3. Model results cover a full unit in rapidity and have been matched to the data at the lowest measured transverse momenta.
TABLE III. Inverse slopes (MeV) partonic.

\begin{tabular}{lccc}
\hline \hline Particle & Rapidity & $\begin{array}{c}\text { Inverse slope } \\
\text { with rescatt }\end{array}$ & $\begin{array}{c}\text { Inverse slope } \\
\text { w/o rescatt }\end{array}$ \\
\hline$\pi$ & 0 & $165 \pm 1$ & $299 \pm 2$ \\
$K$ & & $230 \pm 3$ & $168 \pm 2$ \\
$N$ & & $358 \pm 8$ & $167 \pm 2$ \\
$\pi$ & 3 & $143 \pm 1$ & $196 \pm 1$ \\
$K$ & & $166 \pm 3$ & $141 \pm 3$ \\
$N$ & & $201 \pm 4$ & $150 \pm 3$ \\
\hline \hline
\end{tabular}

agreement between model predictions and data is reasonable, but not as good as for the thermal case; it should be remarked, though, that in the thermal case, a parameter adjustment was made for each particle species. The $p_{t}$ distributions are compared to data in Figs. 14 and 15, where the agreement is good at midrapidity and somewhat inferior near $y=3$. Inverse slopes vary with mass (see also Table III) in a way expected for flow, again without flow appearing explicitly in the calculations. In both cases, $d n / d y$ and transverse spectra, the data are shown for protons rather than nucleons $(p+\bar{p})$, but the conclusions drawn are not affected.

\section{DISCUSSION}

Consider again Fig. 13 which compares $d n / d y$ distributions from the thermal and parton models with rescattering with BRAHMS data. As seen, for $y<3.5$, i.e., the region covered by data, both models reproduce reasonably well the shapes of the distributions, although for pions the parton model does not fall off quite as rapidly as the data for $y>2$. For $y>3.5$ (where there are no data), both models agree with each other for pions, whereas for kaons and protons there is a significant disagreement: the thermal model does not fall off as rapidly as the parton model.

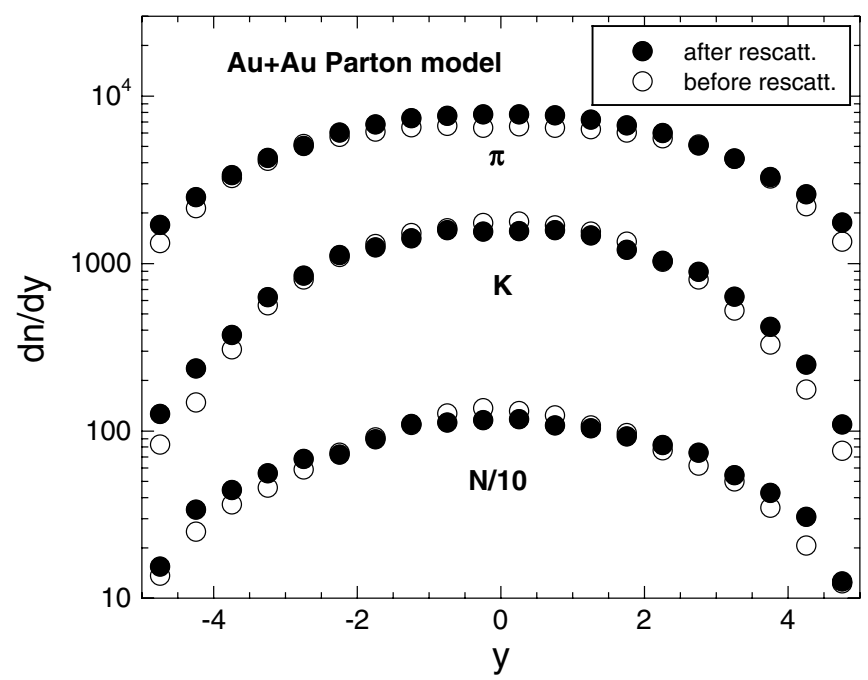

FIG. 12. Rapidity density $d n / d y$ for the cluster model vs c.m. rapidity, before and after rescattering. $N / 10$ stands for nucleon multiplicity divided by 10 . 


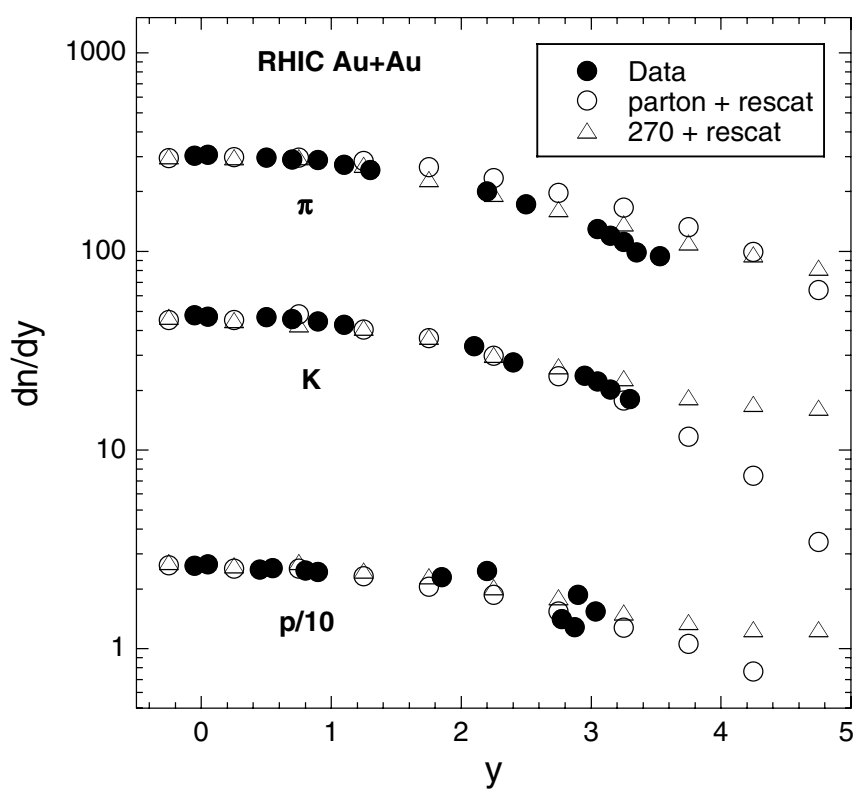

FIG. 13. Rapidity densities vs c.m. rapidity for both partonic and thermal models with rescattering and for data [4,5]. Model results were normalized to the experimental data near $y=0$.

The results for the $m_{t}$ slope parameters and $p_{t}$ distributions are now revisited for both models. As seen in Figs. 8 and 9 and Table II, the thermal model for $T=270 \mathrm{MeV}$ initially gives $m_{t}$ slope parameters for all particles of roughly $300 \mathrm{MeV} / \mathrm{c}$ with those for pions slightly higher than for kaons and nucleons; whereas after rescattering, one sees the flow effect of slope(pion) $<$ slope(kaon) $<$ slope(nucleon). While $m_{t}$ slopes give a qualitative representation of the spectra, a more precise comparison with BRAHMS data is given by directly overlaying the model distributions with data, as shown with $p_{t}$ distributions in Figs. 10 and 11 for the thermal model. As mentioned earlier, the shapes of the data are almost perfectly described by the thermal model with rescattering for the $y=0$ cut and reasonably well reproduced for $y=3$. For the parton model, looking at Table III, the initial $m_{t}$ slope parameters

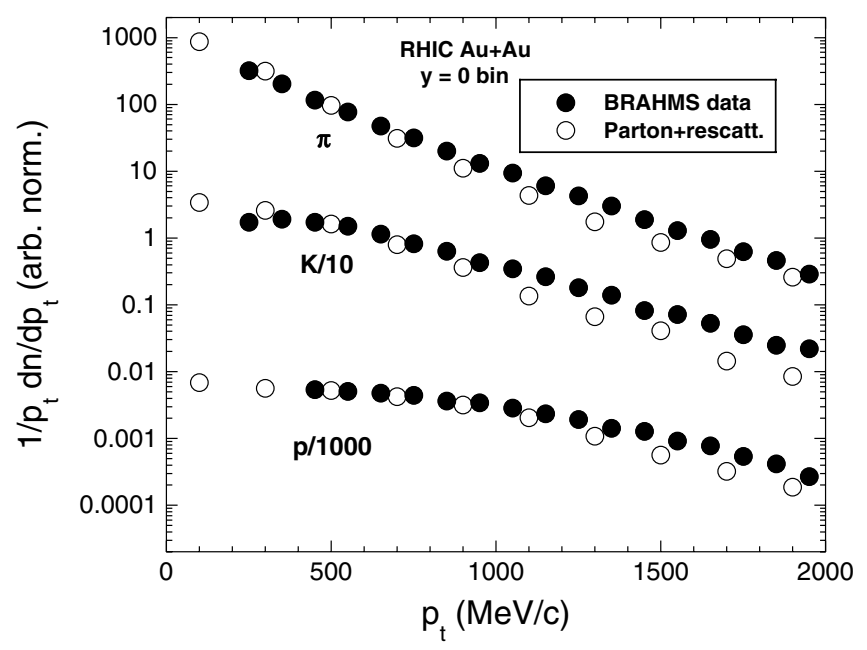

FIG. 14. Same as Fig. 10, but for the parton model.

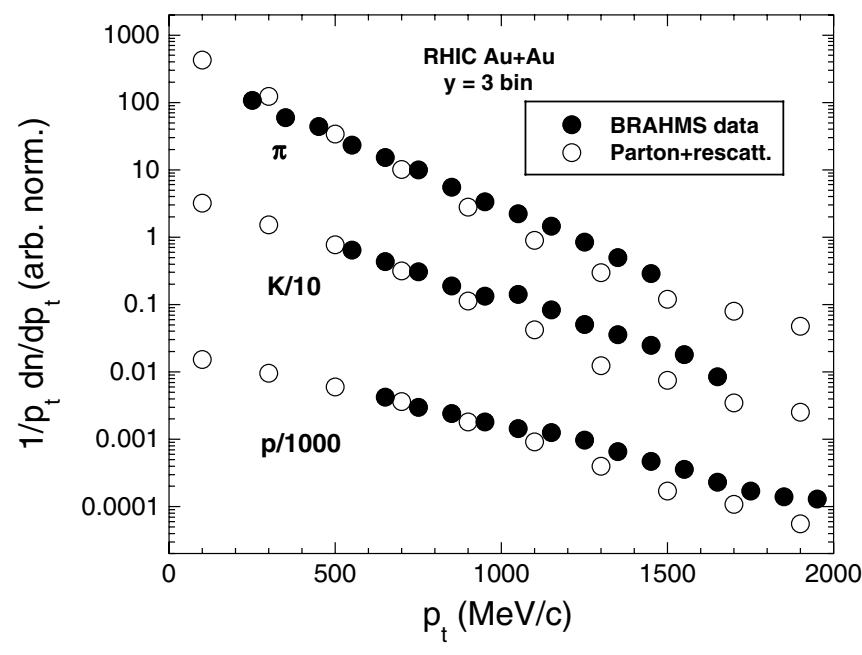

FIG. 15. Same as Fig. 14, but near $y=3$.

are significantly smaller than those for the thermal model (Table II), and the initial kaon and nucleon slopes compared with the pion slopes are fractionally much smaller. After rescattering, the flow effect is produced as for the thermal model but to a lesser extent, as would be expected given the initial slopes for the parton model. This weakened flow effect for the parton model is reflected in the comparison of $p_{t}$ distributions with data shown in Figs. 14 and 15. Although reasonable agreement is seen between the parton model with rescattering and the data for $p_{t}<1000 \mathrm{MeV} / c$, the model systematically falls off faster than data for $p_{t}>1000 \mathrm{MeV} / c$. As with the thermal model, the agreement with data is not as good for the $y=3$ cut as with $y=0$.

The two event-generating models used here are very different and rather schematic. The thermal-like model has no dynamic features and therefore little predictive power, the fitting procedure described in Sec. II C will have to be repeated at each incident energy, and the direct agreement with experiment without rescattering regarding the transverse spectra is rather poor. The partonic model is in principle a dynamic model with predictive power; however, the pion coalescence mechanism is certainly ad hoc and may have to be adjusted at each incident energy. Also, here the predicted transverse spectra agree rather poorly with experiment without rescattering. The main goal in this work has been to study the effects of hadronic rescattering on the hadrons produced by the two different models of the initial stage of the collision. Although the two models are very different, i.e., thermal-like hadrons vs parton clusters, after rescattering they both give qualitatively similar hadronic rapidity, $m_{t}$, and $p_{t}$ distributions, which describe reasonably well results from a RHIC experiment, the thermal model doing somewhat better than the parton model. Although rescattering has only a weak effect on the rapidity distributions for either model, it appears to strongly affect the $m_{t}$ (and $p_{t}$ ) distributions. Even though the two models strongly disagree in the $m_{t}$ distributions they produce for the three particle species, rescattering effects are able to largely wash out these differences such that after rescattering, the particle distributions are now in qualitative agreement. We conclude from this work that features seen 


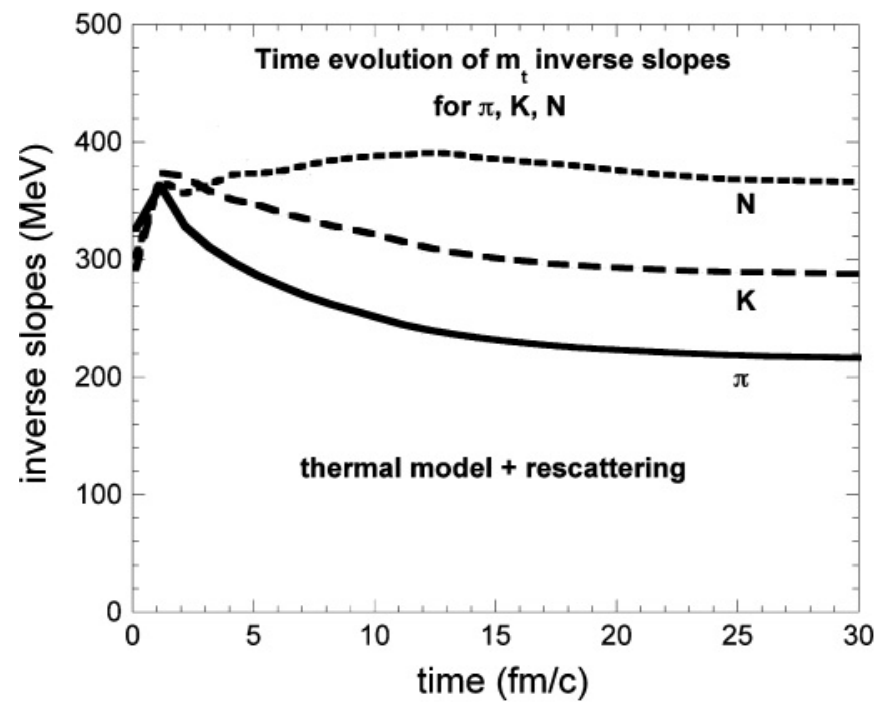

FIG. 16. Inverse slopes with rescattering for the thermal model $(T=270 \mathrm{MeV})$ vs time. Inverse slopes were found from exponential fits to the $m_{t}$ spectra at the various times.

in $m_{t}$ (and $p_{t}$ ) distributions before rescattering are mostly due to the overall temperature scale of the initial stage, and rescattering effects are not very sensitive to the details of the initial stage model used. It is, however, remarkable that rescattering changes the spectra in the same fashion as found in an earlier publication [19] where a third event-generator model was used: "cooling" of the pion spectra and "heating" of the nucleon spectra with the kaons in between.

The two last figures, Figs. 16 and 17, show the inverse slopes for $m_{t}$ spectra from the thermal and the partonic models, respectively, both with rescattering, plotted vs time $t$. The density of particles builds up as $t$ increases from zero until the volume increase with time overtakes the formation of new particles and the density starts to decrease, which happens at about $t=4 \mathrm{fm} / c$. At large $t$, a steady state is reached for the inverse slopes, and the pattern of increasing inverse slope with increasing particle mass, seen from Tables II and III, becomes evident. The changes at small times are very fast, and the pushing of the faster pions on the other particles is clear, while the pions themselves lose momentum. The first $10 \mathrm{fm} / c$ are very important for the development of the final slope pattern. The inverse slopes in the two figures do not

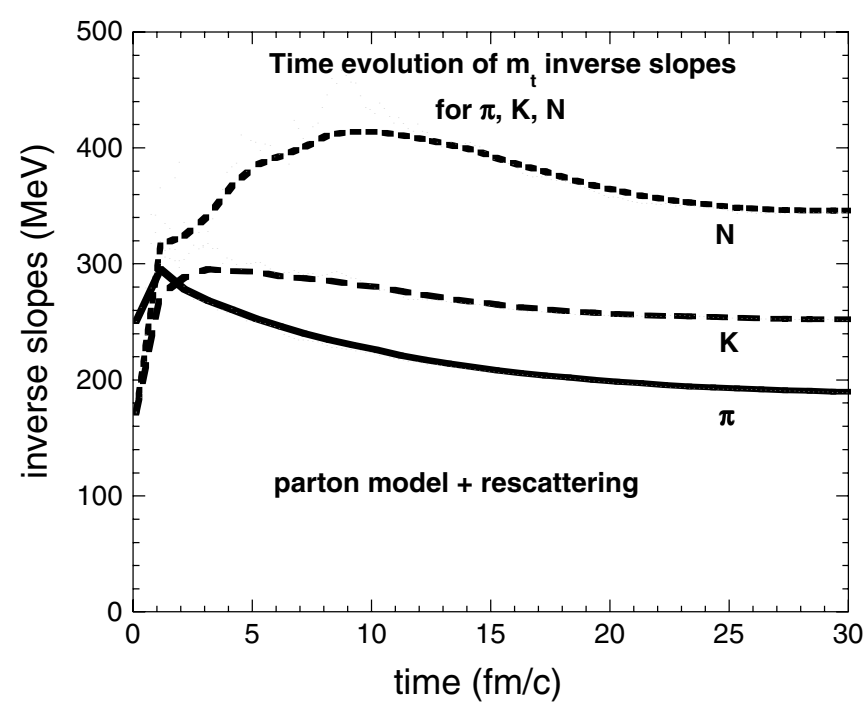

FIG. 17. Same as Fig. 16, but for the partonic model.

agree quantitatively with the numbers in Tables II and III at low $t$ because new particles enter fast as their formation times are reached, while in the tables the spectra are for all particles at freeze-out; moreover, rapidity ranges and $m_{t}$ ranges for the exponential fits are different between the tables and Figs. 16 and 17. The conclusion is, to the extent that the model approaches used here reflect in some reasonable way what happens in the real heavy ion collisions in the laboratory, that one should evidently not draw strong conclusions from the inclusive hadronic $y$ and $m_{t}$ spectra, regarding neither the presence of flow nor the initial collision conditions.

It would be interesting to look at other hadronic observables such as elliptic flow and Hanbury-Brown and Twiss (HBT) interferometry using these two models to see if such observables can be used to better discriminate between initial conditions.

\section{ACKNOWLEDGMENTS}

The authors particularly thank Tracy L. Smith for expert systems management at the Ohio end of the collaboration. The authors also acknowledge financial support from the U.S. National Science Foundation under Grant PHY-0355007 and from the Danish SNF for travel expenses.
[1] T. J. Humanic, Nucl. Phys. A715, 641 (2003).

[2] T. J. Humanic, Int. J. Mod. Phys. E 15, 197 (2006).

[3] T. J. Humanic, Phys. Rev. C 73, 054902 (2006).

[4] I. G. Bearden et al. (BRAHMS Collaboration), Phys. Rev. Lett. 93, 102301 (2004); P. Christiansen, Ph.D. thesis, Niels Bohr Institute, University of Copenhagen (2003).

[5] I. G. Bearden et al. (BRAHMS Collaboration), Phys. Rev. Lett. 94, 162301 (2005); D. Ouerdanne, Ph.D. thesis, Niels Bohr Institute, University of Copenhagen (2004).

[6] J. D. Bjorken, Phys. Rev. D 27, 140 (1983).

[7] P. Braun-Munzinger, D. Magestro, K. Redlich, and J. Stachel, Phys. Lett. B518, 41 (2001); P. Braun-Munzinger, K. Redlich, and J. Stachel, Quark-Gluon Plasma 3, edited by R. C. Hwa and X.-N. Wang (World Scientific, Singapore, 2004), p. 491.

[8] Handbook of Mathematical Functions, edited by M. Abramowitz and I. A. Stegun (Dover, New York, 1965), p. 374 .

[9] From the web address: pdg.lbl.gov/2006/reviews/hadronicrpp. pdf.

[10] R. A. Kers et al. (OPAL Collaboration), Phys. Lett. B320, 417 (1994).

[11] D. H. Perkins, Introduction to High Energy Physics, 3rd. ed. (Addison Wesley, Reading, MA, 1987), p. 295.

[12] B. Alper et al. (R203 Collaboration), Nucl. Phys. B100, 237 (1975); K. Guetler et al. (R803 Collaboration), Phys. 
Lett. B64, 111 (1976); K. Guetler et al. (R803 Collaboration), Nucl. Phys. B116, 77 (1976); C. Albajar et al. (UA1 Collaboration), ibid. B335, 261 (1990).

[13] A. D. Jackson and H. Boggild, Nucl. Phys. A470, 669 (1987).

[14] H. Boggild, Proc. 2nd Workshop Experiments and Detectors for RHIC, Lawrence Berkeley Laboratory, May 25-29, 1987, LBL-24604, p. 302.

[15] X.-N. Wang and M. Gyulassy, Phys. Rev. D 44, 3501 (1991).
[16] I. G. Bearden et al. (BRAHMS Collaboration), Phys. Rev. Lett. 88, 202301 (2002).

[17] M. Herrmann and G. F. Bertsch, Phys. Rev. C 51, 328 (1995).

[18] M. Prakash, M. Prakash, R. Venugopalan, and G. Welke, Phys. Rep. 227, 321 (1993).

[19] T. J. Humanic, Phys. Rev. C 57, 866 (1998).

[20] I. Arsene et al. (BRAHMS Collaboration), Nucl. Phys. A757, 1 (2005). 